\title{
The evaluation of primary care physicians' knowledge on common respiratory diseases: Findings of Action Now Study: an online survey of primary care physicians in China
}

\section{Zihan Pan}

Peking University First Hospital

\section{Ting Yang}

Institute of Respiratory Medicine, Chinese Academy of Medical Science

Chunhua Chi ( $\nabla$ chichunhua2012@qq.com )

Peking University First Hospital

Jiping Liao

Peking University First Hospital

\section{Yahong Chen}

Peking University Third Hospital

\section{Kewu Huang}

Beijing Chao-yang Hospital, Capital Medical University

\section{Chen Wang}

Chinese Academy of Medical Sciences \& Peking Union Medical College

\section{Research article}

Keywords: primary car, knowledge assessment, asthma, COPD, CAP, influenza, electronic questionnaire survey

Posted Date: May 18th, 2020

DOl: https://doi.org/10.21203/rs.3.rs-27479/v1

License: (c) (i) This work is licensed under a Creative Commons Attribution 4.0 International License. Read Full License 


\section{Abstract \\ Background}

Primary care plays an important role in the management of respiratory diseases and primary care physicians' ability is the cornerstone.

\section{Methods}

An e-questionnaire was distributed to attendees of respiratory diseases academic conferences in China from July, 2017 to December, 2018 to assess primary care physicians' knowledge level of asthma, CAP, COPD and influenza in China.

\section{Results}

The feedback rate was $100 \%$, including 4815 valid questionnaires, 3802 (79.0\%) from community hospitals and $1013(21.0 \%)$ from township hospitals. The average score of the questionnaire was $83.31( \pm 20.397)$ and $72.12( \pm 20.898)$ in township and community hospitals $(P \otimes 0.05)$, respectively. $61.4 \%$, $48.7 \%$ and $42.5 \%$ of the primary care physicians were aware of clinical manifestations of COPD, asthma and simple influenza. $85.7 \%, 8.1 \%, 16.1 \%$ and $1.0 \%$ knew how to diagnose COPD, asthma, CAP and influenza, respectively. $94.4 \%$ of the physicians didn't know bronchodilators for COPD; $53.7 \%$ knew nonpharmacological treatments for COPD. $73.6 \%$ were unable to deal with asthma attacks. $65.1 \%$ didn't know what the most essential and important treatment for influenza was. $92 \%$ physicians didn't know the management for stable COPD; 3.0\% knew all prevention and management measures for asthma. 37.9\% knew all the preventive measures for CAP. $44.9 \%$ didn't know the important role of influenza vaccine in preventing influenza and its complications.

\section{Conclusions}

Primary care physicians in China had a poor knowledge on asthma, CAP, COPD and influenza, but township hospitals physicians had a better knowledge than community hospitals physicians.

\section{How This Fits In}

Some sporadic studies have evaluated the knowledge of primary care physicians on chronic obstructive pulmonary disease (COPD) and asthma, but no persuasive studies have assessed their knowledge of influenza and community-acquired pneumonia(CAP) in China. This is the first and largest national evaluation of its kind and evaluated COPD, asthma, influenza and CAP simultaneously. The results will provide a robust reference for the design of a series systematic training courses on respiratory diseases for physicians in primary care in China or similar settings. 


\section{Introduction}

Respiratory diseases are the most common diseases in China ${ }^{[1]}$ and large amount of medical and societal resources have been spent on these diseases. On the one hand, there is a tremendous population of chronic non-communicable respiratory diseases patients. It estimated that there were 99 million patients with chronic obstructive pulmonary disease (COPD $)^{[2]}$ and 43.7 million patients with asthma ${ }^{[3]}$ in China, with an impressed prevalence of $13.7 \%^{[2]}$ and $4.3 \%{ }^{[3]}$, respectively. On the other hand, the incidence and mortality of acute communicable respiratory diseases are also rising yearly in China. The incidence of influenza has increased from $14.9381 / 100,000$ in 2009 to $33.0994 / 100,000$ in 2017; the excess mortality of all causes caused by influenza was $(6.94-17.2) / 100,000^{[4]}$. What's more, influenza virus ranks first among viruses caused community-acquired pneumonia(CAP) ${ }^{[5]}$. In recent years, China has even experienced several out-breaks of influenza and the increasing excess mortality of influenza caused some kind of social panic ${ }^{[6,7]}$.

Although township hospitals are superior medical institutions of community hospitals, both of them are representatives of primary care institutions in China ${ }^{[8]}$. They are the first line in management of respiratory diseases and primary care physicians' ability is the cornerstone. In the fighting against COVID19 , primary care in China have made great contributions, and they can play a more important role in the management of common respiratory diseases. National strategies ${ }^{[9,10]}$ have been released to promote and enhance the development of primary care. Meanwhile, guidelines for management of $\mathrm{CAP}^{[5,11]}$ and influenza ${ }^{[12]}$ in primary care are updated as conditions changing. Furthermore, chronic respiratory diseases' prevention and treatment is one of the major actions of Healthy China 2030 Action Plan ${ }^{[13]}$.

To help strategies fully implemented, the current management ability of primary care physicians on respiratory diseases should be assessed. In order to solve the pivotal problems in management of respiratory diseases in primary care and to optimize the capacity building of primary care physicians, the Chinese Alliance for Respiratory Diseases in Primary Care (CARDPC) was founded in 2015. The role and responsibility of the alliance is to promote the standardized management procedures of common respiratory diseases in primary care by providing systematically training, developing and disseminating guidelines of common respiratory diseases for primary care ${ }^{[14]}$. Since the establishment of the organization, besides annual conferences, CARDPC has undertaken a series of efforts to improve the ability of primary care physicians to manage common respiratory diseases, including Action Now program.

Action Now stands for "Assessment of CAP, asthma, influenza, and COPD knowledge", a national survey conducted from 2017 to 2018 aiming at comprehensively exploring the knowledge of these four common respiratory diseases among primary care physicians in China. An underlying objective is to identify primary care physicians' knowledge weak areas in current primary care practice and use the findings to design further education programs. 


\section{Methods}

The cross-sectional online survey was conducted by electronic questionnaire. A QR-Code and web link of the questionnaire were created by Wenjuanxing, a research consultancy specialising in online research. People visited the questionnaire by scanning the QR-Code via the prominent social APP Wechat or by clicking the web link on laptop. The questionnaire (online supplementary file 1) was administered to attendees of the conferences hold by CARDPC across the country. Participation was by choice of the individual. Attendees who agreed to take part in the survey were only able to complete the questionnaire once under the guidance and supervision of the on-site investigators and then submitted on-line. All respondents were anonymized. Data collection continued from July, 2017 to December, 2018, the questionnaire was available at any time during this time period.

The questionnaire consisted of two parts, demographic characteristics information and knowledge survey questions. Demographic characteristics included gender, age, education level, number of experiences years, current vocational title, specialty, region, and grade of institutions. Knowledge survey questions were assessed using items adapted from the relevant guidelines $[5,12,15,16]$ and focused on four core aspects: (1) epidemiology and clinical characteristics/features; (2) diagnostic criteria; (3) treatment; (4) preventive measures. Open questions were also used to explore their needs of training for the four diseases, and the results would be reported in another paper in the near future.

The questionnaire was developed by a group of respiratory physicians. 534 physicians were invited to pretest the questionnaire which resulted in some adjustments and changes to improve it. The questionnaire comprised 12 questions on COPD, 9 questions on asthma, 8 questions on CAP, 9 questions on influenza, each question has 5 points (Supplementary Box 1). For multi-option questions, respondents can get all the 5 points only when they pick out all the correct answers.

\section{Analysis}

Microsoft Excel and SPSS 17.0 software were used for statistical analysis.

Descriptive statistics were used to present demographic characteristics of respondents. Continuous variables were expressed as the mean \pm standard deviation, and categorical variables were presented as relative frequencies and percentages. And correct rates of individual questions were compared by community and township hospitals using chi-squared statistics. The t-test and $95 \%$ confidence intervals (Cls) were used to assess whether differences in scores between the two different medical institutions were significant. $p$ values less than 0.05 were considered as statistically significant. No imputation was performed for missing data.

\section{Results}

A total of 7391 people attended the conferences hold by CARDPC. Among them, 7353 attendees visited the questionnaire via Wechat and 38 via web link. Overall, 7391 questionnaires were returned with a 100\% 
response rate. Responses were eligible if the respondent was:

- a primary care physician working in community or township hospitals, including General Practitioner, respiratory physician, Traditional Chinese-West medical physicians and internal physician;

- managing respiratory diseases in community or township hospitals; and

- had fully completed the questionnaire.

This resulted in 4815 (65.1\%) eligible responses (Supplementary Figure 1).

\section{Characteristics of respondents}

Overall, $64.6 \%$ respondents were women, $92.8 \%$ respondents were younger than 50 years old. Approximately two thirds (67.0\%) of them had a Bachelor degree. $38.8 \%$ respondents had been in practice for less than 10 years, $47.9 \%$ had an intermediate vocational title, more than half $(65.2 \%)$ of them were specialized in General Practice. $71.1 \%$ respondents practiced in community hospitals. There was an over representation of respondents from north China $(70.3 \%)$ compared to south China $(0.3 \%)$ (Supplementary Table 1).

\section{Knowledge evaluation}

It was clear from the results of the study that the overall knowledge of COPD, asthma, CAP and influenza among respondents was inadequate.

\section{Scores of the questionnaire and each disease}

Overall, the average score of all the 4815 respondents was $74.47( \pm 21.287), 39.2 \%(74.47 / 190)$ of the total score of the questionnaire, $83.31( \pm 20.397)(43.8 \%, 83.31 / 190)$ and $72.12( \pm 20.898)(38.0 \%, 72.12 / 190)$ in township and community hospitals $(P \otimes 0.05)$, respectively. However, either the average score of the whole questionnaire or the average score of each disease was less than $60 \%$. Although, the best part was COPD, with an average score of $53.4 \%(32.04 / 60)$ of its section, the average score of asthma, CAP, and influenza were only $38.2 \%(17.18 / 45), 26.1 \%(10.43 / 40)$, and $32.9 \%(14.82 / 45)$ of the relative parts, respectively. Physicians in township hospitals had higher scores. Scores of diseases were shown in Supplementary Table 2.

\section{Epidemiology and clinical characteristics}

It was disappointing to note that low proportions of respondents were aware of the characteristic symptoms or clinical manifestations of COPD (61.4\%), asthma (48.7\%) and simple influenza (42.5\%). Meanwhile, less than half of them knew the most important cause of COPD (49.2\%), common pathogens of CAP (22.0\%), and the epidemiological characteristics of influenza (16.4\%). This finding was important in view of the fact that, in primary care settings, the above diseases were primarily clinical diagnosed based on epidemiological characteristics, clinical manifestations and signs after the exclusion of other respiratory conditions, such as tuberculosis. 
$85.7 \%$ of the respondents were aware of the diagnosis standard of COPD, however, $45.3 \%$ of them didn't know the criteria of GOLD II COPD. Only $8.1 \%$ of them were aware how to diagnose asthma, meanwhile, only $16.1 \%$ of them were able to correctly make the clinical diagnosis of CAP. Very few respondents were aware of the pathogenic diagnostic specimens of $\operatorname{CAP}(2.1 \%)$, the standardized diagnosis of influenza $(1.0 \%, 46 / 4815)$ and $(0.5 \%, 25 / 4815)$ the diagnostic criteria for severe influenza.

\section{The treatment}

The vast majority (94.4\%) of the respondents didn't correctly choose bronchodilators for COPD; just over one-half of them (53.7\%) knew the non-pharmacological treatments for COPD. Furthermore, $73.6 \%$ of respondents were unable to properly deal with asthma attacks; only $9.4 \%$ of them chosen the right treatment plan for newly diagnosed mild asthma patients. For young adults with no underlying disease, only $2.1 \%$ of respondents correctly selected all of the initial empirical anti-infective therapy for CAP. More than half of respondents $(65.1 \%)$ didn't know what the most essential and important treatment for influenza is.

\section{Preventive measures}

Most respondents (92\%) didn't pick out all the management for stable COPD; nearly one third of them $(27.7 \%)$ knew the duration of daily oxygen inhalation (>15 hours) for long-term home oxygen therapy. Only $3.0 \%$ of respondents selected all prevention and management measures for asthma. Just over one third of respondents (37.9\%) knew all the preventive measures for CAP. For influenza, nearly half of physicians (44.9\%) didn't know the important role of influenza vaccine in preventing influenza and its complications.

Correct rate details of each item in each disease were shown in Supplementary Table 3 and Supplementary Table 4.

\section{Discussion}

\section{Summary}

With the help of the most prominent Chinese social medium Wechat, which made a terrific contribution to the response rate of the survey, this survey allowed more insight into primary care physicians' knowledge on COPD, asthma, CAP and influenza. However, from the results we can see that primary care physicians' knowledge on these four common respiratory diseases was significantly poor. On the whole, either the average score of the questionnaire or the average score of each disease was less than $60 \%$ of the corresponding part. Foremost, physicians can't correctly identify the clinical features of each disease. Only $61.4 \%$ of physicians were aware of the clinical features of COPD, less than half of them knew clinical characteristics of asthma and influenza ( $48.7 \%$ and $42.5 \%$, respectively). What's worse, they knew less about the diagnosis criteria of asthma, CAP and influenza. Only $8.1 \%, 16.1 \%$ and $1.0 \%$ of them can 
make right diagnosis of asthma, CAP and influenza, respectively. Although, it was gratified to see that more than $85 \%$ of the primary care physicians knew how to diagnose COPD, almost half $(45.3 \%)$ of

respondents didn't know the criteria for GOLD II COPD. This was related to a series of policies ${ }^{[17-19]}$ on COPD management which have increased and strengthened the relative training on COPD.

Even though physicians in township hospitals had a better knowledge of these diseases, the knowledge level of influenza between them was similar, which may be due to the incidence of influenza has increased yearly in China so that the government has attached great importance to influenza training. The knowledge of treatment was even worse. Compared with the high correct rate of COPD diagnosis, a very small proportion of physicians were aware of bronchodilators for COPD. As the most two common chronic non-communicable respiratory diseases, only $8 \%$ and $3 \%$ were aware of the management of stable COPD and asthma, respectively.

Above all, physicians at township hospitals had a better knowledge level than community hospital physicians, which provided a possibility for referrals between township hospitals and community hospitals for common respiratory diseases, but a sound knowledge reserve is a prerequisite. From the above, we can see that primary care physicians' knowledge on these common respiratory diseases needs to be greatly improved. However, in view of the outstanding performance of primary care in combating COVID-19 pandemic, we also believe that with high-quality training primary care physicians will manage these diseases very well.

\section{Strengths and limitations}

This is the first and largest study to assess primary care physicians' knowledge on CAP, asthma, influenza, and COPD simultaneously, with such a huge sample, high response rate and broad geographical areas in China. Although only $65.1 \%$ of the questionnaires were analyzed finally, the sample size of the study was still the largest of its kind. Though the sample population was unevenly distributed, it was sufficient to get an overview of the current knowledge level of primary care physicians about these four common respiratory diseases.

However, due to the survey was conducted on conferences, the number of attendees from each area were related to the places of the conferences, which resulted in some areas were overrepresented while some were underrepresented. Thereby the generalizability of the findings for some provinces or cities was limited. For more comprehensive view to present a thorough picture of knowledge level of primary care physicians in specific areas, surveys like this but with more physicians to take part in are needed so as to provide more individualized and targeted training for certain regions.

\section{Comparison with existing literature}

There were studies to investigate primary care physicians' knowledge of COPD and asthma ${ }^{[20-25]}$ around the world, and the conclusions were nearly the same, that is, primary care physicians' knowledge of COPD and asthma were insufficient. Similar studies have also been carried out in China, but in relatively smaller 
sample size and were only regionally based ${ }^{[26,27]}$. Nevertheless, seldom studies focused on knowledge assessment of influenza either in the world or in China. There was one study assessed the knowledge of CAP, but just in one city ${ }^{[28]}$.

\section{Implications for Research and/or practice}

Respiratory diseases account for significant health service and societal costs in China, but the knowledge of common respiratory diseases among primary care physicians is not optimistic. Up to 2017, there were less than 8000 respiratory physicians registered in China ${ }^{[1]}$. However, it's far from enough for these respiratory physicians to manage more than 140 million people with respiratory diseases. It is a crisis time for respiratory diseases management in China and primary care must take action now. Although the current knowledge level of primary care physicians were unsatisfactory, the training programs initiated by CARDPC across the country is going on. Action Now study laid the foundation for the follow-up training programs and future quality improvement work. An updated education program would be designed based on these findings, then a set of courses that are more targeted, practical and more in line with primary care physicians' needs will be launched. Furthermore, under the furtherance of CARDPC, the first guideline of COPD, asthma and CAP management in primary care have been released, and medications for these common respiratory diseases have been introduced to primary care institutions in some areas with the help of CARDPC. Up to the end of 2018, there were 29 branches of CARDPC spread in 29 provinces or municipalities ${ }^{[29]}$ ( Supplementary figure 2).

As a member of the International Primary Care Respiratory Group (IPCRG), CARDPC actively participates in primary care research work and international collaborations on respiratory diseases, and strives to build a comprehensive respiratory diseases management system in primary care settings in China. In the COVID-19 pandemic, CARDPC also played a leading role in the prevention and control in primary care in China. We believe that with the attempts and facilitation of CARDPC, management of respiratory diseases in primary care in China will make great progress in the near future.

\section{Declarations}

Acknowledgements Acknowledge the support from Chinese Thoracic Society (CTS), Chinese Alliance for Respiratory Disease in Primary care (CARDPC) and Soong Ching Ling Foundation for Clinical Research on Respiratory Disease.

Authors' contributions Zihan Pan wrote the paper with input from all other authors. Ting Yang, Jiping Liao, Yahong Chen, Kewu Huang and Chen Wang provided critical review and advice of the manuscript.

Funding This study was funded by the Capital Fund for Development of Health Research (2018-2-4075), CAMS Innovation Fund for Medical Sciences (CIFMS)(2018-I2M-1-001) and National Key R\&D Program of China(2016YFC1303900).

Competing interests The authors have declared no competing interests. 
Ethics approval The study was approved by Peking University First Hospital Research Ethics Committee (REC).

Consent for publication All authors have read and approved the final draft for publication.

Availability of data and materials The datasets used and/or analysed during the current study are available from the corresponding author on reasonable request.

Provenance and peer review Not commissioned; externally peer reviewed.

\section{References}

1. Health Year. Book 2017:P25(in Chinese).

2. Wang $\mathrm{C}, \mathrm{Xu} \mathrm{J}$, Yang L, et al. Prevalence and risk factors of chronic obstructive pulmonary disease in China (the China Pulmonary Health [CPH] study): a national cross-sectional study. Lancet. 2018 Apr 28;391(10131):1706-1717.

3. Huang $\mathrm{K}$, Yang $\mathrm{T}$, Xu J, et al. Prevalence, risk factors, and management of asthma in China: a national cross-sectional study. Lancet. 2019;394(10196):407-18.

4. Li Wen juan,Wang Da yan. Research progress on the disease burden of influenza in China. Chinese Journal of Zoonoses.1-6(in Chinese).

5. Chinese Medical Association, Chinese Medical Journals Publishing House,Chinese Society of General Practice. et al. Guideline of adult community acquired pneumonia for primary care: practice version(2018)Chin J Gen Pract,Feburary,2019. Vol.18,No.2.(in Chinese).

6. Zhang H, Xiong Q, Wu P, Chen Y, NHL L, Cowling BJ. Influenza-associated mortality in Yancheng, China, 2011-15. Influenza Other Respir Viruses. 2018;12(1):98-103.

7. Xu-Xiang Liu Y, Li Y, Zhu. Seasonal pattern of influenza activity in a subtropical city. .

8. Li X, Lu J, Hu S. The primary health-care system in China. Lancet. 2017;390:2584-94.

9. National Guide of the State Council on Establishing a General Practitioner System.July. 2011. http://www.gov.cn/zwgk/2011-07/07/content_1901099.htm.(in Chinese).

10. National guide on Promoting the Hierarchical Diagnosis and (in Chinese)

National guide on Promoting the Hierarchical Diagnosis and. System T.

September,2015.http://www.gov.cn/zhengce/content/2015-09/11/content_10158.htm\#.(in Chinese).

11. Guidelines for the diagnosis and treatment of community-acquired pneumonia in children. (2019 edition). Clinical Education of General Practice Sep.2019,Vol.17,No.9 (in Chinese).

12. National Health Commission of the Peoples' Republic of China. Protocol for diagnosis and treatment of influenza(2018 revised version). Chin J Clin Infect Dis, February 2019,Vol.12,No.1.

13. Healthy China 2030 Action Plan. http://www.gov.cn/xinwen/2019-07/15/content_5409694.htm. (in Chinese). 
14. Chinese Alliance for Respiratory. Diseases in Primary Care. https://www.cardpc.org/.

15. GINA Executive and Science Committee. Global Strategy for asthma management and prevention 2018. http: //www. gina.com.

16. Global Initiative for Chronic Obstructive Lung Disease. Global strategy for the diagnosis, management and prevention of chronic obstructive pulmonary disease 2019. reporthttps://goldcopd.org/wp-content/uploads/2017/02/wms-GOLD-2017-FINAL.pdf.

17. The National Health and Family Planning Commission on Printing and Distributing the Work Plan. (Trial) of Chronic Disease and Nutrition Surveillance for Chinese Residents. (2018-11-26) http://www.gov.cn/xinwen/2014-10/12/content_2763401.htm. (in Chinese).

18. Medium-long term plan of prevention and treatment for chronic diseases. (2017-2025, in Chinese) http://www.gov.cn/xinwen/2017-02/14/content_5167942.htm.(in Chinese).

19. National Notice on Printing and Distributing the. Technical Plan for the Diagnosis and Treatment of Chronic Obstructive Pulmonary Disease. (in Chinese).

20. Öner EF, Köktürk N, Mungan D, et al. Assessing the knowledge in primary health care following an educational course structured in the context of GARD chronic airway diseases national control program. Tuberk Toraks. 2017;65(2):80-9.

21. Hanna Sandelowsky N, Natalishvili, Ingvar Krakau.copd management by Swedish general practitioners-baseline results ot the PRIMAIR study. Scandinavian Journal of Primary Health Care.2018.

22. Göktalay T, Tuncal AN, Sarı S, Köroğlu G, Havlucu Y, Yorgancıoğlu A. Knowledge Level of the Primary Healthcare Providers on Chronic Obstructive Pulmonary Disease and Pulmonary Rehabilitation. Pulm Med. 2015. 2015: 538246.

23. Olivier T, Rutschmanna J-P, Janssensb B, Vermeulenc,et al. Knowledge of guidelines for the management of COPD: a survey of primary care physicians. Respir Med. 2004;98:932-7.

24. Zaurbek Aisanov C, Bai. Otto Bauerle.Primary care physician perceptions on the diagnosis and management of chronic obstructive pulmonary disease in diverse regions of the world. International Journal of COPD 2012:7 271-282.

25. Yawn B, Wollan PC, Textor KB, Yawn RA. Primary care physicians', nurse practitioners' and physician assistants' knowledge, attitudes and beliefs regarding COPD: 2007 to 2014. Chronic Obstr Pulm Dis (Miami). 2016;3(3):628-35.

26. Li F, Cai Y, Zhu Y, et al. The evaluation of general practitioners' awareness/knowledge and adherence to the GOLD guidelines in a Shanghai suburb. Asia Pac J Public Health. 2015;27(2):NP2067-78.

27. Study on knowledge level and Related Factors of Chronic Obstructive. Pulmonary Disease in Primary physicians. Chin Clinic physicians J.2017.45.6:45-50. (in Chinese).

28. Liu Jian Z, Jing C. Qijian. Current status of diagnosis and treatment of community acquired pneumonia in Shanghai revealed by a questionnaire analysis. Chin J Tuberc Respir Dis,April,2018. Vol.41,No.4. (in Chinese). 
29. https://www.cardpc.org/page/show/16.

\section{Supplementary Files}

This is a list of supplementary files associated with this preprint. Click to download.

- FigureS1flowchartofrespondents.docx

- FigureS2branchesofCARDPC.docx

- TableS1Demographiccharacteristicsofrespondents.docx

- Tables2Scoresamongphysiciansoffourdiseases.docx

- TableS3correctrateofitemsofCOPDandasthma.docx

- TableS4correctratesofitemsofCAPandinfluenza.docx

- SupplementaryBox1QuestionnaireofActionNowstudy.docx 